\title{
Isolation and characterization of Burkholderia sp. strain CCA53 exhibiting ligninolytic potential
}

\author{
Hironaga Akita ${ }^{*}$, Zen-ichiro Kimura ${ }^{2}$, Mohd Zulkhairi Mohd Yusoff ${ }^{1,3}$, Nobutaka Nakashima ${ }^{4,5}$ \\ and Tamotsu Hoshino ${ }^{1,4}$
}

\begin{abstract}
Microbial degradation of lignin releases fermentable sugars, effective utilization of which could support biofuel production from lignocellulosic biomass. In the present study, a lignin-degrading bacterium was isolated from leaf soil and identified as Burkholderia sp. based on 165 rRNA gene sequencing. This strain was named CCA53, and its lignindegrading capability was assessed by observing its growth on medium containing alkali lignin or lignin-associated aromatic monomers as the sole carbon source. Alkali lignin and at least eight lignin-associated aromatic monomers supported growth of this strain, and the most effective utilization was observed for $p$-hydroxybenzene monomers. These findings indicate that Burkholderia sp. strain CCA53 has fragmentary activity for lignin degradation.
\end{abstract}

Keywords: $16 \mathrm{~S}$ rRNA gene sequencing, Burkholderia, Lignin-associated aromatic monomer, Lignin-degrading bacterium

\section{Background}

One of the consequences of the increasing population and industrial development is greater fossil fuel consumption and, in turn, greenhouse gas emission, which exacerbates the problem of global warming. It has been proposed that use of biofuels produced from biomass feedstocks could serve as alternatives to fossil fuel that would slow global warming. First-generation biofuels are mostly produced from edible feedstocks such as corn, sugarcane and starch (Ho et al. 2014; Islam et al. 2015). The advantages of edible feedstocks are that they have a high sugar content, require simple sugar extraction methods, and a variety of technologies for biofuel production are available. However, edible feedstocks are also consumed as our food and by our livestock, which means biofuel production competes with production to meet our dietary needs. By contrast, second-generation

\footnotetext{
${ }^{*}$ Correspondence: h-akita@aist.go.jp

${ }^{1}$ Research Institute for Sustainable Chemistry, National Institute of Advanced Industrial Sciences and Technology (AIST), 3-11-32 Kagamiyama, Higashi-Hiroshima, Hiroshima 739-0046, Japan Full list of author information is available at the end of the article
}

biofuels are produced from lignocellulosic biomass, which is usually indigestible (Ho et al. 2014; Islam et al. 2015). Moreover, lignocellulosic biomass is widely distributed and is the most abundantly available raw material on Earth.

Unfortunately, industrial production of second-generation biofuels faces significant obstacles, as the production process is much more laborious than that of first-generation biofuels. The microbial process for converting lignocellulosic biomass to biofuel typically consists of three steps: pretreatment, enzymatic hydrolysis and fermentation. At the pretreatment step, cellulose, hemicellulose and lignin are released through decomposition of the complex structure of lignocellulosic biomass. At the enzymatic hydrolysis step, cellulosic and hemicellulosic polysaccharides are converted into saccharified solution containing mixed sugars. Finally, the saccharified solution is fed to microorganisms as a carbon source in the fermentation step (Ho et al. 2014; Islam et al. 2015). However, lignin is composed of heterogeneous aromatic acids that commonly used industrial host microorganisms such as Saccharomyces cerevisiae and Escherichia coli cannot efficiently 
assimilate. On the contrary, several aromatic acids produced by lignin decomposition cause membrane disruption, enzyme inhibition and DNA damage in microbial cells, ultimately reducing productivity. A few bacteria, including Phanerochaete chrysosporium, Rhodococcus erythropolis and Streptomyces coelicolor, are known to assimilate lignin (Ahmad et al. 2010). However, these microorganisms are not suitable for industrial production of second-generation biofuels, because their culture conditions are intricate and their growth are relatively slow. Here we report screening, isolation and characterization of a lignin-degrading bacterium from leaf soil sample. This bacterium exhibited rapid growth on solid medium containing alkali lignin as the sole carbon source and assimilated lignin-associated aromatic monomers.

\section{Methods}

\section{Isolation of the bacterium strain}

Soil samples were collected from Higashi-Hiroshima City in Hiroshima Prefecture, Japan. An M9 plate (pH 7.2) was used for isolation and contained the following ingredients: $17 \mathrm{~g} \mathrm{~L}^{-1} \mathrm{Na}_{2} \mathrm{HPO}_{4} \cdot 12 \mathrm{H}_{2} \mathrm{O}, 3 \mathrm{~g} \mathrm{~L}^{-1} \mathrm{KH}_{2} \mathrm{PO}_{4}$, $0.5 \mathrm{~g} \mathrm{~L}^{-1} \mathrm{NaCl}, 1 \mathrm{~g} \mathrm{~L}^{-1} \mathrm{NH}_{4} \mathrm{Cl}, 0.24 \mathrm{~g} \mathrm{~L}^{-1} \mathrm{MgSO}_{4} \cdot 7 \mathrm{H}_{2} \mathrm{O}$, $0.011 \mathrm{~g} \mathrm{~L}^{-1} \mathrm{CaCl}_{2} \cdot 2 \mathrm{H}_{2} \mathrm{O}, 15 \mathrm{~g} \mathrm{~L}^{-1}$ agar and $1 \mathrm{~g} \mathrm{~L}^{-1}$ alkali lignin (Tokyo Chemical Industry, Tokyo, Japan). The plate was inoculated with $1 \mathrm{~mL}$ of a $10 \%$ soil wash solution $(\mathrm{w} / \mathrm{v})$ and allowed to grow at $37{ }^{\circ} \mathrm{C}$ for 2 days. After this initial cultivation, a single colony was successively re-streaked on a new M9 plate at least three times to obtain a pure colony. The purified strain was then grown aerobically at $37^{\circ} \mathrm{C}$ in Nutrient Broth (Kyokuto, Tokyo, Japan) and preserved at $-20{ }^{\circ} \mathrm{C}$ as a suspension in Nutrient Broth supplemented with glycerol (20\%, $\mathrm{w} / \mathrm{v})$.

\section{S rRNA gene amplification and sequencing}

Genomic DNA from the isolated microorganism was extracted using an illustra bacteria genomicPrep Mini Spin Kit according to manufacturer's instructions (GE Healthcare, Buckinghamshire, UK) and then used as the template for $16 \mathrm{~S}$ rRNA gene amplification. 16S rRNA gene was amplified using KOD-plus-DNA polymerase (TOYOBO, Osaka, Japan) with bacterial universal primers 27f (Lane 1991) and 1391r (Turner et al. 1999). After purifying the amplified PCR products using Wizard SV Gel and PCR Clean-Up System (Promega, Madison, WI, USA), they were cloned into pTA2 vector (TOYOBO).

Sequencing was performed using universal primers M13 forward (5'-GTAAAACGACGGCCAGT-3') and M13 reverse (5'-CAGGAAACAGCTATGAC-3') on an Applied Biosystems model 3730xl DNA Analyzer at Fasmac (Kanagawa, Japan).

\section{Characterization of ligninolytic activity}

The ligninolytic activity of the isolated microorganism was assessed by monitoring the increase in $\mathrm{OD}_{600}$, which reflected the cell growth. The $\mathrm{OD}_{600}$ was measured by monitoring the difference between the cell and cell-free turbidity values using an Eppendorf BioSpectrometer (Eppendorf, Hamburg, Germany). The isolated microorganism was pregrown overnight using Nutrient Broth (Kyokuto, Tokyo, Japan) and then diluted 3:100 with fresh M9 medium ( $\mathrm{pH}$ 7.2) containing $1 \mathrm{~g} \mathrm{~L}^{-1}$ alkali lignin or $5 \mathrm{mM}$ lignin-associated aromatic monomers.

\section{Results and discussion}

\section{Screening of lignin-degrading bacteria}

Microbial degradation of lignin has been primarily studied in white-rot fungi, which are capable of producing several extracellular ligninolytic enzymes, including laccase, lignin peroxidase, manganese peroxidase and versatile peroxidase (Dashtban et al. 2010). Brown-rot fungi are also known to degrade lignin using Fenton-based free radicals (Arantes et al. 2012). Bacterial degradation of lignin has been studied much less (Ahmad et al. 2010; Bugg et al. 2011). A few bacterial species belonging to the genera Arthrobacter, Pseudomonas, Sphingobium, Streptomyces and Rhodococcus have been shown to have ligninolytic activity in vitro (Bugg et al. 2011). Although these species have several favorable features, including high growth potential, amenability to well developed genetic techniques and ease of practical utilization, their lignin degradation capabilities are much lower than those of fungi. We therefore screened for lignin-degrading bacteria with a high capacity for lignin degradation.

To obtain lignin-degrading bacteria, filtrates were prepared from several soil samples and then plated onto M9 plates $(\mathrm{pH}$ 7.2) containing alkali lignin as the sole carbon source. When the plate was incubated aerobically at $37{ }^{\circ} \mathrm{C}$, individual colonies were obtained from the leaf soil filtrate. However, except a single colony, individual colonies were not obtained after standard dilution plating onto another M9 plate. Thus, the single colony was obtained and named strain CCA53. When cultured in M9 liquid medium, strain CCA53 exhibited cell growth that depended on lignin assimilation (Fig. 1); a two-fold increase in $\mathrm{OD}_{600}$ was observed over the first $8 \mathrm{~h}$, but it remained unchanged thereafter. On the other hand, strain CCA53 showed no-growth in the absence of alkali lignin.

\section{Phylogenetic analysis}

To identify the phylogeny of strain CCA53, the 16S rRNA gene sequence was determined (1303 bp; accession number: LC107951). On the basis of $16 \mathrm{~S}$ rRNA gene sequence homology, the closest relatives were determined to be Burkholderia multivorans strain LMG $13010^{\mathrm{T}}$ (99.7 \%), B. 


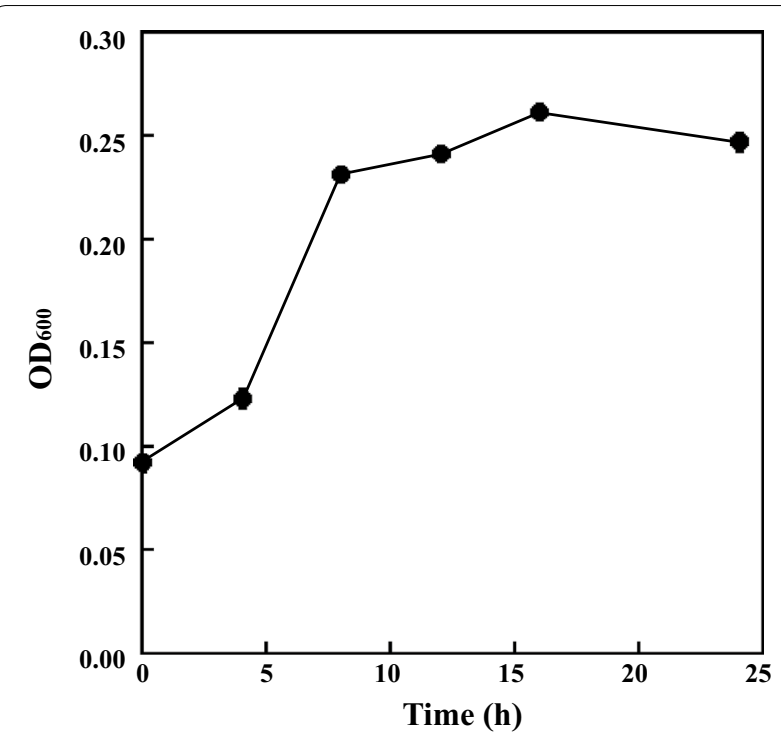

Fig. 1 Growth of Burkholderia sp. strain CCA53. Culture was performed in M9 liquid medium containing alkali lignin as the sole carbon source. Error bars indicate SE $(n=3)$

latens strain R-5630 (99.4\%), B. cenocepacia strain LMG $16656^{\mathrm{T}}$ (99.2\%), and with somewhat lower sequence homology, Pandoraea apista strain LMG $16407^{\mathrm{T}}(95.1 \%)$. In the neighbor-joining phylogenetic tree (Fig. 2), strain
CCA53 fell inside the cluster comprising members of the genus Burkholderia. Thus, strain CCA53 was identified as Burkholderia sp. (strain number: HUT-8135).

Burkholderia is a genus of the class $\beta$-proteobacteria and is separated from the former Pseudomonas rRNA gene homology group II (Yabuuchi et al. 1992). This genus was originally proposed by Yabuuchi et al. (1992) and more than 80 Burkholderia species have been reported to date. Burkholderia species have been classified into two major clusters and several subgroups through phylogenetic analyses of the $16 \mathrm{~S}$ rRNA gene, $\operatorname{rec} A, \operatorname{gyr} B$, $r p o B$ and $a c d S$ gene sequences as well as their genome sequences (Estrada-de los Santos et al. 2013). Group A comprises plant-associated species and saprophytic species. For example, B. mimosarum, B. nodosa, B. sabiae, $B$. tuberum and $B$. phymatum can facilitate nitrogen fixation in legumes (Suárez-Moreno et al. 2012), and B. phytofirmans and $B$. unamae are able to promote plant growth via the activity of 1-aminocyclopropane-1-carboxylate deaminase (Suárez-Moreno et al. 2012). In addition, $B$. kururiensis, B. phenoliruptrix, B. sartisoli, B. unamae and $B$. xenovorans all exhibit abilities to degrade several lignin-associated aromatic monomers and are used for decontamination of polluted soils (Suárez-Moreno et al. 2012), as well as for agricultural purposes such as phytoremediation and biocontrol. On the other hand, group B contains opportunistic pathogens that infect animals,

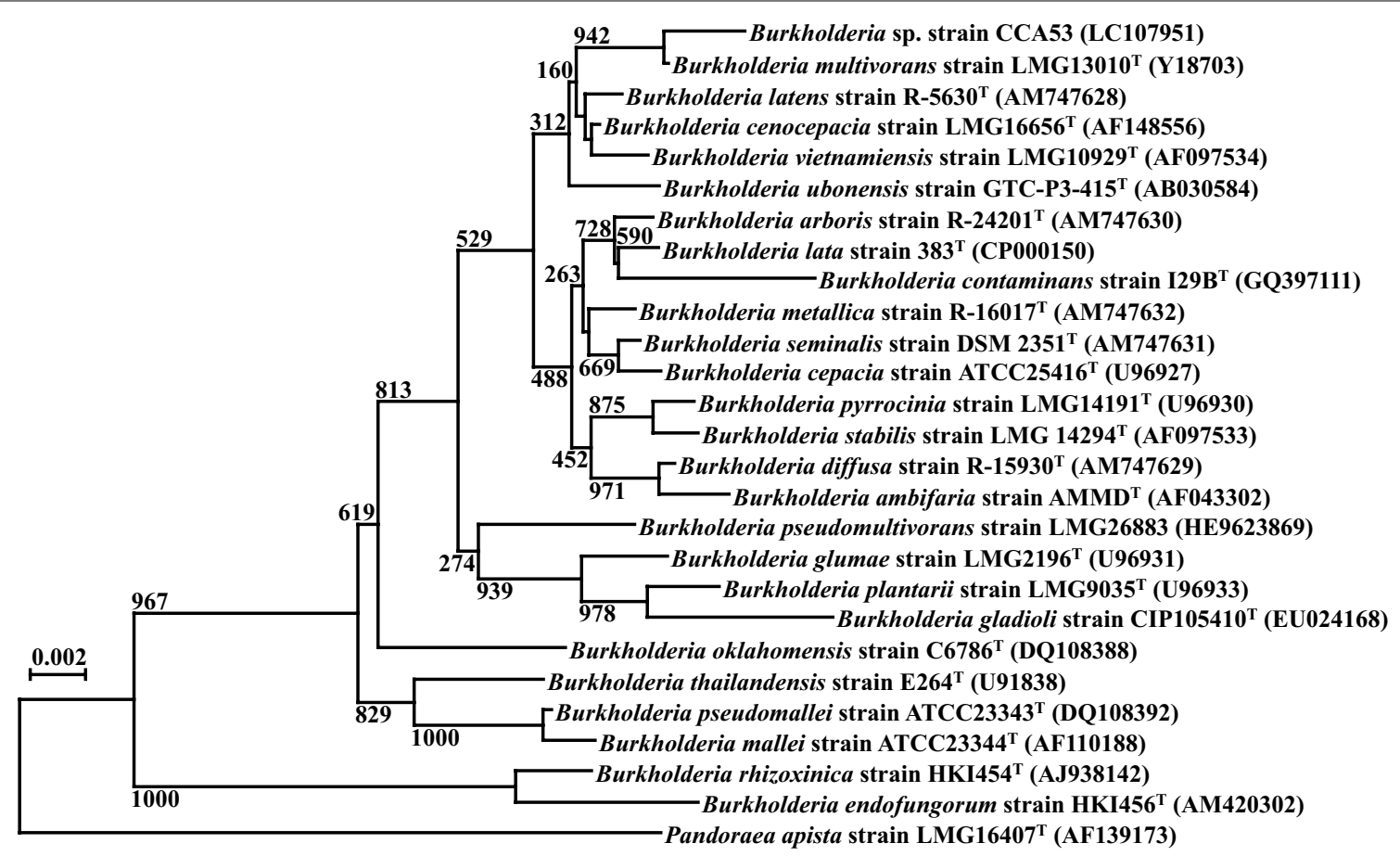

Fig. 2 Phylogenetic tree reconstruction based on 16S rRNA gene sequences. All sequences were compared with reference 16S rRNA gene sequences available in the GenBank/EMBL/DDBJ databases using BLAST. Multiple alignment and construction of neighbor-joining phylogenetic tree (Saitou and Nei 1987) were performed using CLUSTAL W version 1.83 (Thompson et al. 1994) 
humans and plants. When the phylogenetic relationships of B. multivorans, B. latens and B. cenocepacia were confirmed, their closest relatives were included in group B. Indeed, $B$. multivorans, $B$. latens and $B$. cenocepacia all have an ability to infect cystic fibrosis patients, which leads to pneumonic illness with fever and respiratory failure (Horsley et al. 2011; Jones et al. 2004). However, these species were recently used as biocatalysts for degradation of aromatic polycyclic hydrocarbons, esters and steroid analogs. Non-aqueous phase liquids containing naphthalene, $n$-hexadecane, $n$-octadecane, $n$-nonadecane, 1-methylnaphthalene and pyrene are degraded by B. multivorans (NG1) with Triton X-100 or rhamnolipid JBR-515 (Mohanty and Mukherji 2013). Two kinds of $p$-hydroxybenzoic acid esters, methyl paraben and propyl paraben, are degraded by B. latens (Amin et al. 2010). Ezetimibe, a selective inhibitor of acyl-coenzyme A, was produced from reduction of 1-(4-fluorophenyl)-5-(2oxo-4-phenyl-oxazolidin-3-yl)-pentane-1,5-dione in culture medium with B. cenocepacia (Singh et al. 2009). It was thus anticipated that Burkholderia sp. strain CCA53 would have the capacity to degrade aromatic acids.

\section{Utilization of lignin-associated aromatic monomers}

The main building blocks of lignin are $p$-hydroxybenzene, guaiacyl (4-alkyl-2-methoxyphenol) and syringyl (4-alkyl-2,5-dimethoxyphenol) units, which are crosslinked by $\mathrm{C}-\mathrm{C}$ bonds (e.g., $5-5, \beta-1, \beta-5, \beta-\beta$ ) and $\mathrm{C}-\mathrm{O}-\mathrm{C}$ bonds (e.g., 4-O-5, $\alpha-\mathrm{O}-4, \beta-\mathrm{O}-4)$ (Vanholme et al. 2010; Zhu et al. 2014). In particular, the $\beta-\mathrm{O}-4$ bond is the most frequent inter-unit linkage, accounting for more than $50 \%$ of all linkages (Santos et al. 2013).

As described above, when cultured using alkali lignin as sole carbon source, Burkholderia sp. strain CCA53 grew inefficiently (Fig. 1). This indicates that this strain has little ability to depolymerize lignin, but it was expected to have an alternative capacity to utilize lignin degradation products. Therefore, to further evaluate the lignindegrading activity of Burkholderia sp. strain CCA53, we assessed its ability to assimilate lignin-associated aromatic monomers. We found that Burkholderia sp. strain CCA53 assimilated eight lignin-associated aromatic monomers (Fig. 3). In particular, strong growth improvements were observed with $p$-hydroxybenzene monomers such as 4-hydroxybenzaldehyde and 4-hydroxybenzoic acid. Benzaldehyde, benzoic acid, catechol and vanillin were also assimilated. By contrast, anisole, $o$-cresol, guaiacol, phenol, syringaldehyde, syringic acid, vanillic acid, vanillyl alcohol and veratryl alcohol were not assimilated. Although Burkholderia sp. strain CCA53 was able to utilize $p$-hydroxybenzene units, only inefficient growth was observed when lignin was fed as a sole carbon source (Fig. 1). These results imply that Burkholderia sp. strain

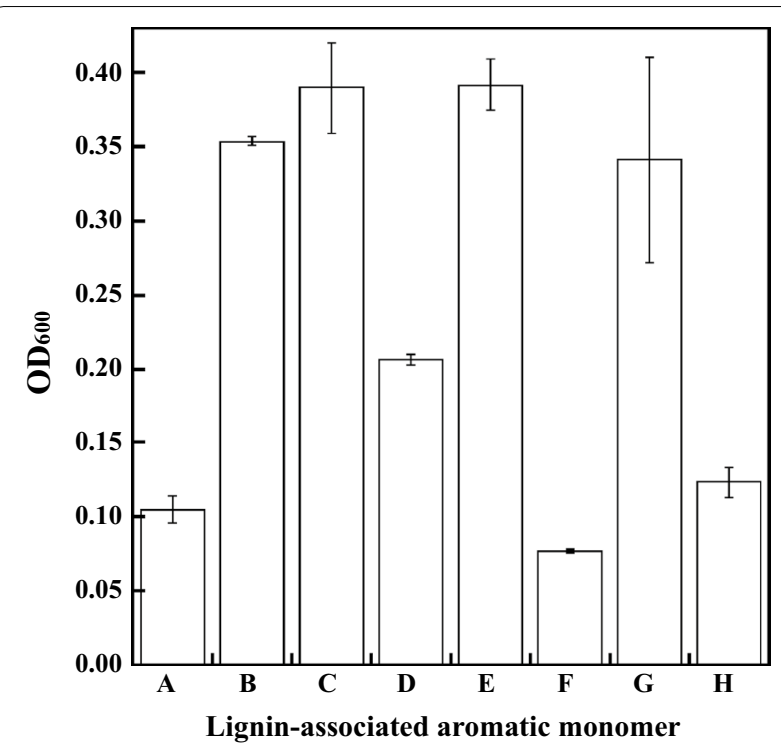

Fig. 3 Growth of Burkholderia sp. strain CCA53 on lignin-associated aromatic monomers. Growth of Burkholderia sp. strain CCA53 in M9 medium containing $5 \mathrm{mM}$ lignin-associated aromatic monomer as the sole carbon and energy source. Experiments were performed in triplicate; A Benzaldehyde, B Benzoic acid, C Catechol, D 4-Hydroxy benzaldehyde, E 4-Hydroxy benzoic acid, F4-Hydroxybenzyl alcohol, $G$ Syringol, $H$ Vanillin. Error bars indicate $\operatorname{SE}(n=3)$

CCA53 lacks activities needed to decompose crosslinks between the main building blocks and to utilize guaiacyl and syringyl units. Consequently, Burkholderia sp. strain CCA53 showed inefficient growth.

Several other Burkholderia species have also shown lignin-degrading capability (Bugg et al. 2011; Kato et al. 1998; Woo et al. 2014). For example, B. cepacia KK01 showed an ability to efficiently assimilate the lignin-associated aromatic monomers $o$-cresol, guaiacol, phenol and vanillic acid, but the assimilation mechanisms have been largely unexplored (Kato et al. 1998). In addition, evidence indicates that the unique lignin-degrading capability of Burkholderia sp. strain LIG30 reflects the expression of two genes predicted to encode multi-copper oxidases and 22 genes encoding putative catalases or peroxidases (Woo et al. 2014). These observations suggest that different Burkholderia species utilize different assimilation mechanisms; thus Burkholderia sp. strain CCA53 may use a specific pathway for degradation of $p$-hydroxybenzene monomers. Such a degradation pathway for 4-nitrobemzoate, one of the $p$-hydroxybenzene monomers, has been proposed in B. cepacia (Peres et al. 2001). To evaluate in detail the capability of Burkholderia sp. strain CCA53 for lignin degradation, we now plan to sequence the organism's entire genome and to implement proteome analysis. We anticipate that these studies will elucidate the lignin utilization pathway of Burkholderia sp. strain CCA53. 


\section{Conclusion}

In this study, we screened lignin-degrading bacteria and the objective bacterium was isolated from leaf soil. Based on 16S rRNA gene sequencing and phylogenetic analysis, the bacterium was identified as Burkholderia sp. strain CCA53. Burkholderia sp. strain CCA53 demonstrated growth on alkali lignin, although growth was rather poor. The capability to utilize lignin-associated aromatic monomers was also relatively limited. The most effective utilization was observed for $p$-hydroxybenzene monomers. The results obtained in this study indicate that Burkholderia sp. strain CCA53 has fragmentary activity for lignin degradation.

\begin{abstract}
Authors' contributions
HA designed this study, performed experiments, participated in the interpretation of the results and drafted the manuscript. ZK and MZMY participated in the design and coordination of this study and helped to revise the manuscript. NN and TH conceived and designed this study, coordinated the experiments, interpreted the results and revised the manuscript for important intellectual content. All authors read and approved the final manuscript.
\end{abstract}

\section{Author details}

${ }^{1}$ Research Institute for Sustainable Chemistry, National Institute of Advanced Industrial Sciences and Technology (AIST), 3-11-32 Kagamiyama, Higashi-Hiroshima, Hiroshima 739-0046, Japan. ${ }^{2}$ Department of Civil and Environmental Engineering, National Institute of Technology, Kure College, 2-2-11 Aga-minami, Kure, Hiroshima 737-8506, Japan. ${ }^{3}$ Department of Bioprocess Technology, Faculty of Biotechnology and Biomolecular Sciences, Universiti Putra Malaysia, 43400 Serdang, Selangor, Malaysia. ${ }^{4}$ Bioproduction Research Institute, National Institute of Advanced Industrial Sciences and Technology (AIST), 2-17-2-1 Tsukisamu-Higashi, Toyohira-ku, Sapporo 062-8517, Japan. ${ }^{5}$ Department of Biological Information, Graduate School of Bioscience and Biotechnology, Tokyo Institute of Technology, 2-12-1-M6-5 Ookayama, Meguro-ku, Tokyo 152-8550, Japan.

\section{Acknowledgements}

We are grateful to all members of the Bio-conversion Research Group at our Institute [Research Institute for Sustainable Chemistry, National Institute of Advanced Industrial Sciences and Technology (AIST)] for their technical assistance and valuable discussion. This work was supported in part by the Science and Technology Research Partnership for Sustainable Development (SATREPS), under Japan Science and Technology Agency (JST) and Japan International Cooperation Agency (JICA).

\section{Competing interests}

The authors declare that they have no competing interests.

Received: 1 February 2016 Accepted: 26 April 2016

Published online: 11 May 2016

\section{References}

Ahmad M, Taylor CR, Pink D, Burton K, Eastwood D, Bending GD, Bugg TD (2010) Development of novel assays for lignin degradation: comparative analysis of bacterial and fungal lignin degraders. Mol BioSyst 6:815-821

Amin A, Chauhan S, Dare M, Bansal AK (2010) Degradation of parabens by Pseudomonas beteli and Burkholderia latens. Eur J Pharm Biopharm 75:206-212

Arantes V, Jellison J, Goodell B (2012) Peculiarities of brown-rot fungi and biochemical Fenton reaction with regard to their potential as a model for bioprocessing biomass. Appl Microbiol Biotechnol 94:323-338
Bugg TD, Ahmad M, Hardiman EM, Singh R (2011) The emerging role for bacteria in lignin degradation and bio-product formation. Curr Opin Biotechnol 22:394-400

Dashtban M, Schraft H, Syed TA, Qin W (2010) Fungal biodegradation and enzymatic modification of lignin. Int J Biochem Mol Biol 1:36-50

Estrada-de los Santos P, Vinuesa P, Martínez-Aguilar L, Hirsch AM, CaballeroMellado J (2013) Phylogenetic analysis of Burkholderia species by multilocus sequence analysis. Curr Microbiol 67:51-60

Ho DP, Ngo HH, Guo W (2014) A mini review on renewable sources for biofuel. Bioresour Technol 169:742-749

Horsley A, Perry C, Martin K, Webb K, Turton J, Kenna D, Jones A (2011) Burkholderia latens infection in cystic fibrosis. J Cyst Fibros 10:291-292

Islam ZU, Zhisheng Y, el Hassan B, Dongdong C, Hongxun Z (2015) Microbial conversion of pyrolytic products to biofuels: a novel and sustainable approach toward second-generation biofuels. J Ind Microbiol Biotechnol 42:1557-1579

Jones AM, Dodd ME, Govan JR, Barcus V, Doherty CJ, Morris J, Webb AK (2004) Burkholderia cenocepacia and Burkholderia multivorans: influence on survival in cystic fibrosis. Thorax 59:948-951

Kato K, Kozaki S, Sakuranaga M (1998) Degradation of lignin compounds by bacteria from termite guts. Biotechnol Lett 20:459-462

Lane DJ (1991) 16S/23S rRNA sequencing. In: Stackebrandt E, Goodfellow M (eds) Nucleic acid techniques in bacterial systematics. Wiley, Chichester, pp 115-147

Mohanty S, Mukherji S (2013) Surfactant aided biodegradation of NAPLs by Burkholderia multivorans: comparison between Triton X-100 and rhamnolipid JBR-515. Colloids Surf B Biointerfaces 102:644-652

Peres CM, Russ R, Lenke H, Agathos SN (2001) Biodegradation of 4-nitrobenzoate, 4-aminobenzoate and their mixtures: new strains, unusual metabolites and insights into pathway regulation. FEMS Microbiol Ecol 37:151-159

Saitou N, Nei M (1987) The neighbor-joining method: a new method for reconstructing phylogenetic trees. Mol Biol Evol 4:406-425

Santos R, Hart PW, Jameel H, Chang H (2013) Wood based lignin reactions important to the biorefinery and pulp and paper industries. BioResources 8:1456-1477

Singh A, Basit A, Banerjee UC (2009) Burkholderia cenocepacia: a new biocatalyst for efficient bioreduction of ezetimibe intermediate. J Ind Microbiol Biotechnol 36:1369-1374

Suárez-Moreno ZR, Caballero-Mellado J, Coutinho BG, Mendonça-Previato $L$, James EK, Venturi V (2012) Common features of environmental and potentially beneficial plant-associated Burkholderia. Microb Ecol 63:249-266

Thompson JD, Higgins DG, Gibson TJ (1994) CLUSTAL W: improving the sensitivity of progressive multiple sequence alignment through sequence weighting, position-specific gap penalties and weight matrix choice. Nucleic Acids Res 22:4673-4680

Turner S, Pryer KM, Miao VP, Palmer JD (1999) Investigating deep phylogenetic relationships among cyanobacteria and plastids by small subunit rRNA sequence analysis. J Eukaryot Microbiol 46:327-338

Vanholme R, Demedts B, Morreel K, Ralph J, Boerjan W (2010) Lignin biosynthesis and structure. Plant Physiol 153:895-905

Woo HL, Utturkar S, Klingeman D, Simmons BA, DeAngelis KM, Brown SD, Hazen TC (2014) Draft genome sequence of the lignin-degrading Burkholderia sp. strain LIG30, isolated from wet tropical forest soil. Genome Announc 2:e00637-14

Yabuuchi E, Kosako Y, Oyaizu H, Yano I, Hotta H, Hashimoto Y, Ezaki T, Arakawa M (1992) Proposal of Burkholderia gen. nov. and transfer of seven species of the genus Pseudomonas homology group II to the new genus, with the type species Burkholderia cepacia (Palleroni and Holmes 1981) comb. nov. Microbiol Immunol 36:1251-1275

Zhu H, Chen Y, Qin T, Wang L, Tang Y, Sun Y, Wan P (2014) Lignin depolymerization via an integrated approach of anode oxidation and electro-generated $\mathrm{H}_{2} \mathrm{O}_{2}$ oxidation. $\mathrm{RSC}$ Adv 4:6232-6238 